\title{
Electron pressure balance in the SOL through the transition to detachment
}

\author{
A.G. McLean ${ }^{\mathrm{a}^{*}}$, A.W. Leonard ${ }^{\mathrm{b}}$, M.A. Makowski ${ }^{\mathrm{b}}$, M. Groth ${ }^{\mathrm{c}}$, S.L. Allen ${ }^{\mathrm{a}}$, J.A. Boedo ${ }^{\mathrm{d}}$, \\ B.D. Bray ${ }^{\mathrm{b}}$, A.R. Briesemeister ${ }^{\mathrm{e}}$, T.N. Carlstrom ${ }^{\mathrm{a}}$, D. Eldon ${ }^{\mathrm{d}}$, M.E. Fenstermacher ${ }^{\mathrm{a}}$, \\ D.N. Hill ${ }^{\mathrm{a}}$, C.J. Lasnier ${ }^{\mathrm{a}}$, C. Liu ${ }^{\mathrm{b}}$, T.H. Osborne ${ }^{\mathrm{b}}$, T.W. Petrie ${ }^{\mathrm{b}}$, V.A. Soukhanovskii ${ }^{\mathrm{a}}$,

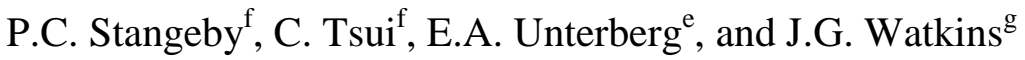 \\ ${ }^{a}$ Lawrence Livermore National Laboratory, Livermore, CA 94550, USA \\ ${ }^{b}$ General Atomics, P.O. Box 85608, San Diego, California 92186-5608 USA \\ 'Aalto University, Espoo, 02150 Finland \\ ${ }^{d}$ University of California San Diego, 9500 Gilman Drive, La Jolla, California, 92093 USA \\ 'Oak Ridge National Laboratory, P.O. Box 2008, Oak Ridge, Tennessee, 37831 USA \\ ${ }^{f}$ University of Toronto Institute for Aerospace Studies, Toronto, M3H 5 T6 Canada \\ ${ }^{g}$ Sandia National Laboratories, P.O. Box 5800, Albuquerque, New Mexico, 87185 USA
}

\begin{abstract}
Upgrades to core and divertor Thomson scattering (DTS) diagnostics at DIII-D have provided measurements of electron pressure profiles in the lower divertor from attached- to fully-detached divertor plasma conditions. Detailed, multistep sequences of discharges with increasing line-averaged density were run at several levels of $P_{i n j}$. Strike point sweeping allowed 2D divertor characterization using DTS optimized to measure $T_{e}$ down to $0.5 \mathrm{eV}$. The ionization front at the onset of detachment is found to move upwards in a controlled manner consistent with the indication that scrape-off layer parallel power flux is converted from conducted to convective heat transport. Measurements of $n_{e}, T_{e}$ and $p_{e}$ in the divertor versus $L_{\text {parallel }}$ demonstrate a rapid transition from $T_{e} \geq 15 \mathrm{eV}$ to $\leq 3 \mathrm{eV}$ occurring both at the outer strike point and upstream of the X-point. These observations provide a strong benchmark for ongoing modelling of divertor detachment for existing and future tokamak devices.
\end{abstract}


Abstract length (150 words): 166 currently

PSI-21 keywords: DIII-D, Fusion reactor blanket, divertor, magnet

$P A C S:$ 52.55.Fa, $52.55 . \mathrm{Rk}, 52.70 . \mathrm{Kz}$

*Corresponding author address: Adam McLean, c/o General Atomics, MS 13-355, P.O. Box

85608, San Diego, California 92186-5608, USA

*Corresponding author e-mail: $\underline{\text { mclean @fusion.gat.com }}$ 


\section{Introduction}

Limited lifetime due to wall erosion and damage to plasma-facing surfaces due to extreme power deposition ( $>10 \mathrm{MW} / \mathrm{m}^{2}$ in steady state) are major impediments to next step fusion devices, even for the most resilient materials (graphite, tungsten). As a solution, a high density 'detached' divertor was developed using incremental gas fuelling in which parallel momentum is transferred efficiently by charge exchange collisions from the plasma to the divertor targets via neutral recycling atoms, not by energetic ions accelerated through the plasma sheath [1]. This regime of detachment is associated with a) reduced plasma pressure, b) significant ionization and impurity radiation losses, and ultimately c) volume recombination in the divertor. Together, these properties lead to a higher radiative power exhaust fraction and reduced incident heat flux to the divertor targets, resulting in less damage and longer wall component lifetimes. For future, high power fusion devices like ITER, operation with a detached divertor will be a necessity. Unfortunately, however, accurate interpretive simulation of observed plasma properties in detachment has remained elusive to date [2], leading to reduced confidence in predictive modelling for ITER. The need to improve modelling quality leads to the need for high quality experimental data characterizing the transition from a divertor where the ion flux in the scrape-off layer (SOL) is 'attached' to the plasma-facing targets to one of detachment at increasing line averaged densities.

Thomson scattering is an ideal diagnostic to perform this task because it provides a passive point measurement (not line-integrated) in the plasma volume and requires minimal interpretation. At DIII-D, the unique divertor Thomson scattering (DTS) diagnostic [3] has recently been refurbished and upgraded to include an improved 7-channel filter set extending the $T_{\mathrm{e}}$ range with error $<10 \%$ from $0.5 \mathrm{eV}-5.0 \mathrm{keV}$, with polarizers to reduce stray light. 
These improvements resulted in the highest accuracy and precision achieved to date, especially at low $T_{\mathrm{e}}$ characteristic of detachment $(<2 \mathrm{eV})$. Additionally, the main plasma core/SOL Thomson system has been upgraded to include a high density array throughout the plasma edge [4]. This enhancement makes it possible to identify the separatrix location and pedestal parameters with high accuracy, parameterize target conditions against pedestal parameters and gradients at the midplane separatrix, and test models describing how conditions in the main SOL influence those at the divertor target.

In this paper experiments carried out to elucidate the roles of different physical processes related to the electron population (in contrast to the ions, which are not measured in the divertor) in the transition from divertor attachment to detachment are described. Enhanced capabilities of the primarily diagnostics, notably the DTS system, and verification of the target sweeping technique in the open DIII-D lower divertor are demonstrated. Subsequent analysis is presented revealing important observations relating to the characteristics of the transition to detachment. These characteristics include a rapid transition in divertor conditions and divergence away from the scaling of target pressure being equal to $1 / 2$ the pressure at the upstream separatrix at detachment onset, and the formation of a pressure hill near the X-point through the high-recycling to fully detached conditions.

\section{Experiment and observations}

DTS was employed in a series of dedicated experiments in which plasmas with increasing line-averaged electron density, $\overline{\boldsymbol{r}_{e}}$, and fractions of the Greenwald density $\left(n / n_{\mathrm{GW}}\right)$ limit were run in edge localized mode (ELM)ing, H-mode. Several levels of injected power $\left(P_{\mathrm{inj}}=2.5,5.5\right.$ and 9.5 MW) were used and $\overline{\tilde{R}_{e}}$ increased in successive discharges until full detachment was reached; this involved a 6-8 step shot sequence at each level of $P_{i n j}$. The magnetic X-point and 
outer strike point (OSP) were swept across the lower floor in order for the plasma to pass across the 8-channel vertical DTS array (Fig. 1). Sweeping of the strike points is a common method to extend OD measurements to 1D and 2D in DIII-D to exploit the open lower divertor. Integrity of plasma conditions at the target as the plasma was swept across the lower divertor floor and shelf is verified by observation of overlapping deuterium (D) and carbon (C) filtered emission spectroscopy [5] (Fig. 2) and Langmuir probe saturation current $\left(I_{\mathrm{sat}}\right)$ when mapped to magnetic flux surfaces $\left(\Psi_{n}\right)$ through the sweep. Inter-ELM data from the DTS taken in time are mapped back to a single representative magnetic equilibrium shape applying the method described in Ref. [6]. This technique allows construction of a 2D profile of $T_{\mathrm{e}}, n_{\mathrm{e}}$, and electron pressure $\left(p_{\mathrm{e}}=T_{e} \cdot n_{e}\right)$ (Fig. 3), and the ability to characterize plasma conditions versus the parallel connection length, $L_{\text {par }}$, throughout the divertor (Fig. 4). Results of the study are shown in Fig. 3 at the extremes of the density scan (increasing left to right), each in H-mode plasmas with $P_{\text {inj }}=2.5 \mathrm{MW}$. In attachment, typical $T_{\mathrm{e}}$ of $20-30 \mathrm{eV}$, and $n_{\mathrm{e}}$ of $2-4 \times 10^{19} / \mathrm{m}^{3}$ are measured at the lower floor. As $n / n_{\mathrm{GW}}$ is increased, recycling at the target increases rapidly (as measured with filterscopes, visible/near infrared spectroscopy, and tangential TV imaging), initially leading to a higher pressure along the full length of the outer leg and a pressure hill near the X-point [Fig. 4(c)], then, as $\overline{n_{e}}$ is raised further, a subsequent decrease below the level in attachment. Once $T_{\mathrm{e}}$ at the target drops to $\lesssim 2 \mathrm{eV}$ and $n_{\mathrm{e}}$ rises to $>1 \times 10^{20} / \mathrm{m}^{3}$ concurrently with the formation of a region of high density below the X-point [7], the ionization front is observed to move upwards off the floor and ultimately beyond the X-point in a controlled manner with increasing $\overline{r_{\overparen{e}}}$. A large recombination region is formed below the X-point with $T_{\mathrm{e}} \leq 1 \mathrm{eV}$ and $n_{\mathrm{e}}$ as high as $1 \times 10^{21} / \mathrm{m}^{3}$ at the highest level of $P_{\text {inj. }}$. These observations are consistent with a transition to radiative loss primarily by convection ( $>50 \%$ ) for parallel heat flux at $T_{\mathrm{e}} \lesssim 15 \mathrm{eV}$, and exclusive transport by convection in radiative, partially detached divertor (PDD) conditions with 
$T_{\mathrm{e}}<5 \mathrm{eV}[6,8]$. This indicates a slowing of the plasma flow approaching the surface in detachment caused by ion-neutral frictional drag through the ionization front. The low temperatures present $(\leq 1 \mathrm{eV})$ also suggest a significant cooling role for molecular species from the fuel $\left(D_{2}\right)$ via molecular assisted recombination and the impurities present [2]. While excluded from subsequent analysis, ELMs were found to 'burn through' the detached region of the outer leg causing excursions in the $n_{\mathrm{e}}$ and $T_{\mathrm{e}}$ and momentary re-attachment except in all but the very highest densities. In all cases, an electron overpressure was observed above the X-point, consistent with similar previous observations in Ohmically heated plasmas [9]. Inter-ELM plasma conditions upstream were mapped to kinetic EFITs and processed using best local fitting of various functional forms through a limited range of magnetic flux $\left(\Psi_{n}=0.995-1.010\right)[10]$ and the results found to have significant correlations with those at the OSP; in Fig. $5, T_{e, O S P}$ is plotted against $n_{e, s e p}, p_{e, O S P}$ Vs. $p_{e, s e p}$, and the heat flux width mapped to the midplane, $\lambda_{q, O S P}$, is plotted against $T_{e, O S P}$. We note that confinement quality at intermediate $P_{\text {inj,NBI}}=5.5 \mathrm{MW}$ is found to remain robust and the $\mathrm{H}$-mode pedestal response to increasing density well reproduced with EPED [11].

\section{Discussion}

The data presented allows exploration of physics relevant to detachment. For each level of $P_{i n j}, T_{e, O S P}$ is found to fall systematically with increasing $n_{e, s e p}$ to the point where partial detachment begins wherein an abrupt drop in $T_{e, O S P}$ is measured (Fig. 5a). The $n_{e, s e p}$ at which a PDD originates increases approximately as $\sqrt{P_{i n i}}$. The value of $T_{e, O S P}$ at which a PDD commences appears to increase with $P_{i n j}$, as does the width of the region in $n_{e, s e p}$ space between the point before and after $T_{e, O S P}$ collapses within the coarse scan of $\overline{n_{e}}$ carried out. This implies that a relatively small change in upstream $n_{e, s e p}$, i.e., $\delta n_{e} / n_{e}<10 \%$, is associated with a rapid 
transition in divertor regime. Similarly, $p_{e, O S P}$ is found to follow a trend of scaling with $1 / 2 p_{e, s e p}$ up to the point where the target ceases to be attached (Fig. 5b), consistent with pressure balance and sonic flow in the SOL [8]. This feature almost scales linearly with $P_{i n j}$, and $p_{e, O S P}$ is found to drop by up to $2-3 \mathrm{X}$. at the transition. No obvious trend is found for $p_{e, O S P}$ after full detachment is reached, however that may be a consequence of limited data at the highest levels of $\overline{\mathrm{r}_{\boldsymbol{c}}}$. Analysis of $\lambda_{q, O S P}$ measured by infrared camera mapped to the midplane shows a sudden 2-4X broadening of the width with detachment onset (Fig. 5c).

Evidence of such a rapid transition in plasma conditions through the transition to divertor detachment seen previously on JET [12] is evident in the dataset here. At the target, the transition from $T_{\mathrm{e}, \mathrm{OSP}} \sim 15-20 \mathrm{eV}$ to that of $T_{\mathrm{e}} \leq 3 \mathrm{eV}$ occurs with very little obvious operating space between those conditions. This is also true of conditions upstream from the target, from $L_{\mathrm{par}} \sim 5-12 \mathrm{~m}$ where, from $n / n_{\mathrm{GW}}=0.83$ to 0.87 , the $T_{\mathrm{e}}$ is found to decrease, and $n_{\mathrm{e}}$ increases rapidly. These observations suggest that without fine control of such a PDD condition it may be challenging to avoid dithering between conditions.

The 2D $p_{\mathrm{e}}$ profile in attached [Fig. 3(c)] and detached divertor [Fig. 3(d)] conditions demonstrate the loss of parallel pressure conservation in the SOL and an $\sim 2-3 \mathrm{X}$ reduction through the region just outboard of the separatrix extending from $L_{\mathrm{par}} \sim 3 \mathrm{~m}$ down to the target in detachment, but simultaneous broadening of the radial $p_{\text {e }}$ profile into the SOL $\left(\Psi_{n} \sim 1.004\right.$ and outboard) compared to the attached divertor case. In contrast to a significant increase in $p_{\mathrm{e}}$ in PDD plasmas $\left(n / n_{\mathrm{GW}} \sim 0.7-0.8\right)$ along most of the upper portion of the divertor leg [Fig. 4(c), $\left.L_{\mathrm{par}} \sim 3-9 \mathrm{~m}\right)$, the $p_{\mathrm{e}}$ profile along this region in the most detached plasmas is similar in both attached and fully detached conditions.

\section{Conclusions and future work}


Electron temperature is the dominant physical parameter controlling divertor detachment [1]. For this reason, regular, reliable measurement of the $2 \mathrm{D}$ profiles of $T_{\mathrm{e}}$, and $n_{\mathrm{e}}$ in the divertor through the transition to detachment is a powerful tool for understanding the processes at play in detachment onset. Experiments at DIII-D discussed here have been carried out at incrementally increasing $\overline{\mathrm{i}_{e}}$ and different levels of $P_{i n j}$ with strike point sweeping in order to provide a fully $2 \mathrm{D}$ picture of divertor conditions through the transition from attached to fully detached divertor operation using the newly upgraded DTS system. Data parameterizing detachment characteristics against plasma conditions at the magnetic separatrix using the high resolution core TS system reveal a delicate balance of power and particle flux at the point of detachment onset, not just at the target itself but also along flux tubes throughout the X-point and divertor leg regions where a rapid transition to low $T_{e}$ is found to occur at increasing $n_{e, s e p}$ with larger $P_{i n j}$. These data provide the basis for comparison of divertor and upstream data using, for example, the basic and extended two point model to determine how well inclusion of simple pressure balance and conduction can predict conditions at the outer target.

These results provide strong constraints on modelling that is now underway for the transition to detached divertor operation [13]. Additionally, future dedicated experiments are planned that explore the influence of variable $I_{\mathrm{p}}$ and $B_{\mathrm{T}}$ to the degree and onset of detachment.

\section{Acknowledgements}

This work was supported in part under the auspices of the US Department of Energy (DOE) by LLNL under DE-AC52-07NA27344 and by the US DOE under DE-FC02-04ER54698, DE-FG02-07ER54917, DE-AC05-00OR22725, and DE-AC04-94AL85000. DIII-D data shown in this paper can be obtained in digital format by following the links at https://fusion.gat.com/global/D3D_DMP. 


\section{References}

[1] A. Loarte, et al., Nucl. Fusion 38 (1998) 331.

[2] M. Wischmeier, M. Groth, A. Kallenback, et al., J. Nucl. Mater. 390-391 (2009) 250.

[3] T.N. Carlstrom, C.L. Hsieh, R. Stockdale, et al., Rev. Sci. Instrum. 68 (1997) 1195.

[4] D. Eldon, B.D. Bray, T.M. Deterly et al., Rev. Sci. Instrum. 83 (2012) 10E343.

[5] R.J. Colchin, D.L. Hillis, R. Maingi, et al., Rev. Sci. Instrum. 74 (2003) 2068.

[6] A.W. Leonard, M.A. Mahdavi, C.J. Lasnier, et al., Nucl. Fusion 52 (2012) 063015.

[7] T.W. Petrie, S.L. Allen, T.N. Carlstrom, et al., J. Nucl. Mater. 241-243 (1997) 639.

[8] A.W. Leonard, M.A. Mahdavi, S.L. Allen, et al., Phys. Rev. Let. 78 (1997) 4769.

[9] M.J. Schaffer, J.A. Boedo, and R.A. Moyer, J. Nucl. Mater. 266-269 (1999) 264.

[10] M.A. Makowski, C.J. Lasnier, A.W. Leonard, et al., these proceedings (2014).

[11] A.W. Leonard, M.A. Makowski, A.G. McLean, et al., these proceedings (2014).

[12] M. Groth, et al, "Poloidal distribution of recycling source and core fuelling in DIII-D, AUG, and JET L-mode plasmas,” $38^{\text {th }}$ EPS, Strasbourg, France (2011).

[13] J.M. Canik, A. Briesemeister, C.J. Lasnier, et al., these proceedings (2014). 


\section{Figure Captions}

Fig. 1. The plasma equilibrium and observation regions by key diagnostics applied to the detachment scan are shown. The swept extent of the strike points across the lower divertor plasma-facing surfaces to provide 2D mapped DTS data is indicated by the three overlaid equilibrium solutions shown.

Fig. 2. Filtered emission diagnostic ('filterscope', or FS) data for $D_{\alpha}, 656 \mathrm{~nm}$ (top row) and CIII, $465 \mathrm{~nm}$ (bottom row) at separate radial chord locations ('FSxxDa'/'FSxxC3') are shown mapped to R-R $\mathrm{R}_{\text {sep }}$ coordinates at the inner target (left column) and outer target (right column). Overlap of the mapped data infer consistent target conditions as the plasma is swept across the lower divertor floor.

Fig. 3. 2D mapped DTS data are shown for a low density discharge with 2.5 MW of NBI power and an attached divertor (left panels) and similarly in a high density discharge with detached divertor (right panels) are shown. Maps of $T_{\mathrm{e}}$ are on the top row, while $p_{\mathrm{e}}$ is shown on the bottom panels for the same discharges. Individual data points are shown as small boxes (each 0.4-0.5 $\mathrm{cm}$ in $\mathrm{R}, 0.9-1.2 \mathrm{~cm}$ in $\mathrm{Z}$ ), while a spatial fit to the data is shown on a regular grid on a rainbow color scale. Equilibrium flux surfaces in the SOL are shown in steps of $0.004 \Psi$.

Fig. 4. Profiles of fitted $T_{\mathrm{e}}$ (a), $n_{\mathrm{e}}$ (b), and $p_{\mathrm{e}}$ (c) for the $P_{\text {inj }}=2.5 \mathrm{MW}$ series of H-mode discharges versus $L_{\text {parallel }}$ are shown, averaged over the flux surface range of $\Psi_{n}=1.000-1.004$. Vertical bars represent the location of the X-point $\left(L_{\mathrm{par}}=7-9 \mathrm{~m}\right)$ and outboard midplane (OMP, $\left.L_{\mathrm{par}}=23-26 \mathrm{~m}\right)$.

Fig. 5. DTS data parameterized against upstream TS data for $\overline{\mathrm{r}_{e}}$ scans at $P_{i n j}=2.5,5.5$, and 9.5 MW. In a) the region where $T_{e, O S P}$ transitions rapidly from a value $\geq 15 \mathrm{eV}$ to $\geq 3 \mathrm{eV}$ is shown in 
yellow. In b) where $P_{e, O S P}=1 / 2 P_{e, \text { sep }}$ is shown as a dashed diagonal line. In c) the heat flux width measured at the midplane is plotted against $T_{e, O S P}$, and the rapid transition region is again shown. 


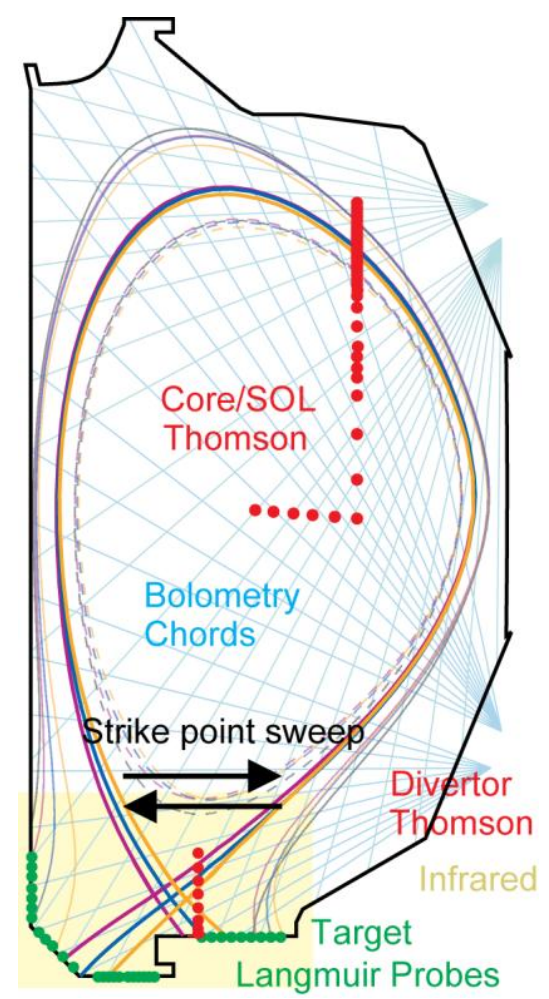

Figure 1 

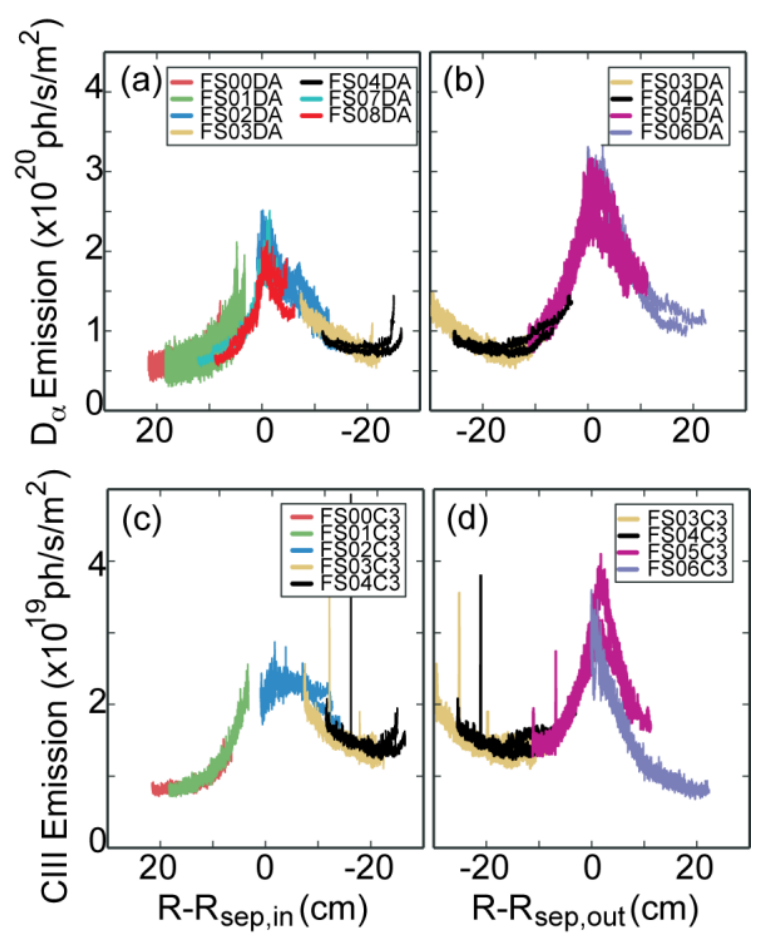

Figure 2 


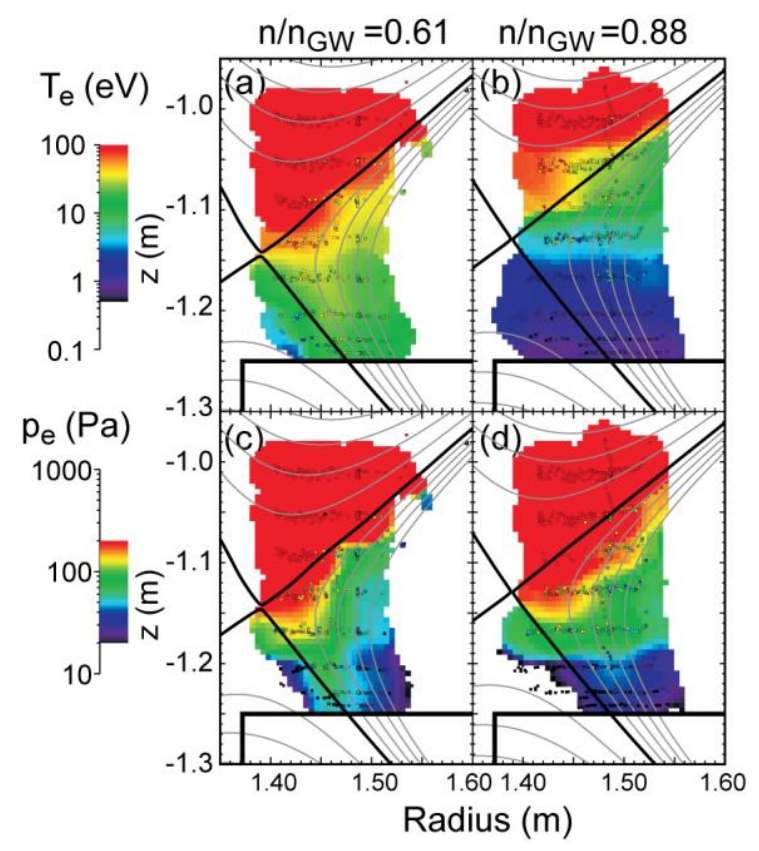

Figure 3 


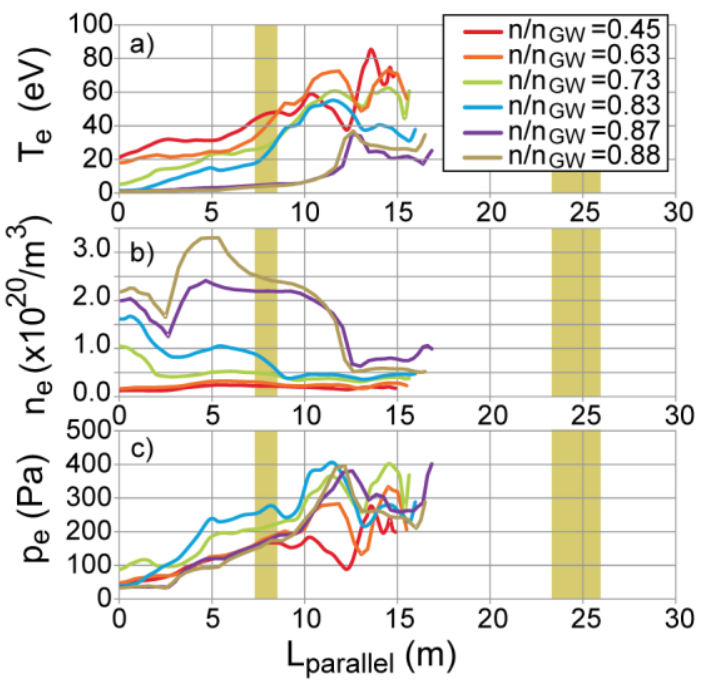

Figure 4 

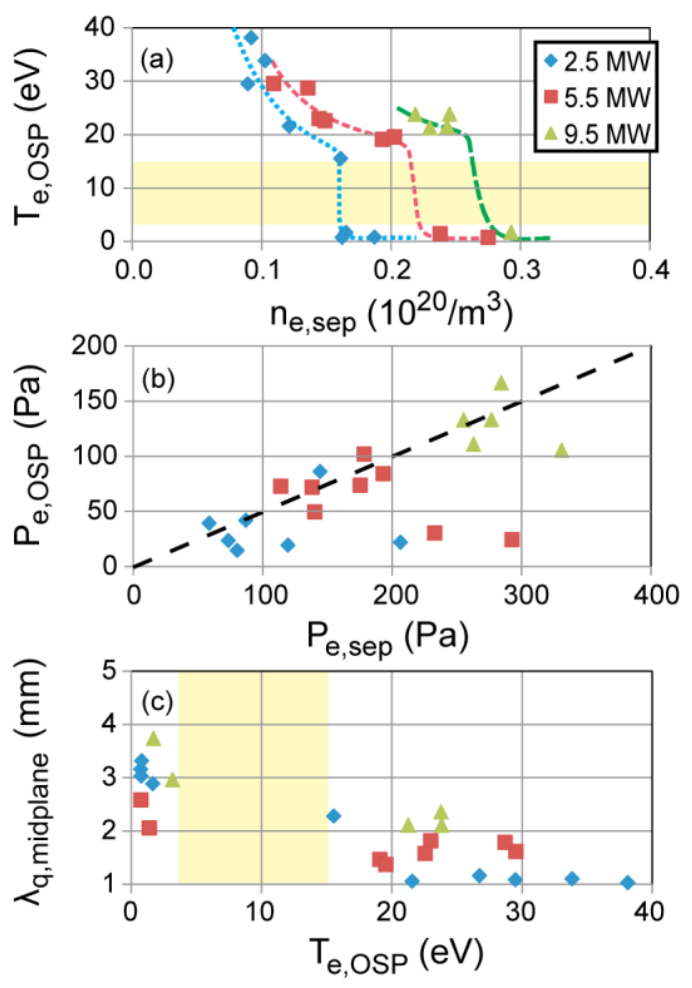

Figure 5 